REGARDS

SUR L'ECONOMIE ALLEMAND

BULLETIN ECONOMIQUE DU CIRAC
Regards sur l'économie allemande

Bulletin économique du CIRAC

$90 \mid 2009$

Varia

\title{
Le chômage partiel, amortisseur social de la crise ?
}

\section{Werner Eichhorst et Paul Marx}

Traducteur : Isabelle Bourgeois et Solène Hazouard

\section{OpenEdition}

\section{Journals}

Édition électronique

URL : http://journals.openedition.org/rea/3580

DOI : $10.4000 /$ rea.3580

ISBN : 978-2-8218-0876-8

ISSN : 1965-0787

Éditeur

CIRAC

Édition imprimée

Date de publication : 1 mars 2009

Pagination : 23-30

ISSN : 1156-8992

\section{Référence électronique}

Werner Eichhorst et Paul Marx, « Le chômage partiel, amortisseur social de la crise ? », Regards sur

l'économie allemande [En ligne], 90 | mars 2009, mis en ligne le 01 mars 2011, consulté le 01 mai 2019. URL : http://journals.openedition.org/rea/3580 ; DOI : 10.4000/rea.3580 


\title{
Le chômage partiel, amortisseur social de la crise?
}

\author{
Werner Eichhorst, Paul Marx
}

Des deux côtés du Rhin, face à la crise, on redécouvre un mécanisme longtemps tombé en désuétude : le chômage partiel. Si on y recourait naguère pour accompagner socialement les restructurations rendues nécessaires par une baisse de l'activité ou forcées par les mutations structurelles de l'économie, il avait largement perdu son attrait. Instrument social pour gérer la réactivité requise dans le domaine de la production industrielle en réponse aux fluctuations des commandes, il avait fini par pâtir d'une certaine rigidité de ses procédures en comparaison de la flexibilité accrue apportée par un droit du travail en pleine évolution en France comme en Allemagne (avec, par exemple, la montée en puissance des comptes épargne-temps).

Or aujourd'hui, le régime du chômage technique prend des allures de panacée dans la gestion sociale de la crise. Les pouvoirs publics français l'ont remis sur le devant de la scène dans le cadre du volet social du plan de relance de l'économie et du soutien à l'industrie automobile, assouplissant la durée des conventions de chômage technique et accroissant la part du financement public des aides aux entreprises. Le gouvernement fédéral, dans le cadre de ses programmes conjoncturels, en a lui aussi accru la durée, la portant de 12 à 18 mois, quel que soit le secteur d'activité, mais accordé la priorité à la formation durant la période d'inactivité forcée. Si dans nos deux pays, le chômage partiel - dénommé " activité partielle » (Kurzarbeit) outre-Rhin - vise le maintien des salariés dans l'emploi, les approches française et allemande diffèrent quelque peu quant à son régime et à son financement, les transferts versés aux bénéficiaires allemands - les salariés exclusivement - étant financés par les seules cotisations sociales. Mais dans les deux cas, et plus encore en Allemagne où les entreprises ont développé dès l'automne 2008, avant même que le régime du chômage partiel soit modifié pour les deux ans à venir, un engouement marqué pour cette forme de contrat de travail qui leur permet de conserver leurs compétences, se pose aussi la question du coût à terme de la généralisation de ce dispositif. Celle du coût social d'abord : sa généralisation ne risque-t-elle pas de privilégier les 'insiders' et d'accroître ainsi durablement les disparités ? Celle du coût budgétaire ensuite : qu'elle soit financée par les cotisations ou par les impôts, ne risque-t-elle pas de creuser encore plus les déficits publics sans pour autant réduire significativement le risque que la crise fait peser sur l'emploi ? (IB)

\section{Un régime généreux d'activité partielle en Allemagne}

En France, lorsqu'une entreprise se voit contrainte de procéder à une réduction du temps de travail ou à un arrêt de production temporaire, les salariés bénéficient de la mesure de "chômage partiel » appelé aussi " chômage technique ". Elle ouvre droit à l'indemnisation du salarié concerné, prise en charge par l'employeur qui s'en voit rembourser une partie par l'Etat. Depuis janvier 2009, l'employeur français verse à ses salariés $60 \%$ de la rémunération horaire initiale brute (contre $50 \%$ auparavant), c'est-à-dire au moins 6,84 € de l'heure. L'Etat lui reverse en contrepartie $3,33 €$ (entreprises de plus de 250 salariés) ou $3,84 €$ (entreprises à effectif inférieur) ; de la sorte, l'Etat finance près de la moitié de l'indemnité sur les revenus les plus modestes. Jusqu'à la fin 2008, l'indemnisation

Le chômage partiel est cofinancé par l'Etat en France 
En Allemagne, il relève de l'assurance chômage du chômage partiel était limitée à 600 heures par salarié sur une période de 4 semaines au maximum. Depuis le début de l'année 2009, ce plafond est porté à 800 heures (voire 1000 heures pour l'industrie automobile et ses sous-traitants) pour une période ne devant pas excéder six semaines consécutives.

En Allemagne, le chômage partiel (Kurzarbeit) présente une organisation différente. Son indemnisation, globalement plus généreuse, est une prestation versée au titre du régime général de l'assurance chômage, et donc financée par les cotisations sociales. Les employeurs ou les secteurs d'activité qui recourent le plus largement au chômage partiel ne sont donc soumis à aucun prélèvement particulier. II existe trois régimes d'indemnisation différenciés: les indemnités de " chômage technique de transfert " (Transferkurzarbeitergeld) dans le cas de la restructuration des d'entreprises, celles de "chômage partiel saisonnier " (Saisonkurzarbeitergeld) spécifique au BTP, et les indemnités de "chômage technique conjoncturel » (konjunkturelles Kurzarbeitergeld : Kug). C'est sur ce dernier régime que se concentre actuellement l'attention.

\section{L'indemnité de chômage partiel conjoncturel}

Le contrat de travail et les obligations de l'employeur sont maintenues

Pour les heures chômées, le salarié est indemnisé ...

...et l'employeur reste soumis aux charges patronales

Assouplissement transitoire du régime Kug pour 2009/2010
Régie par le chapitre III du Code social allemand (Sozialgesetzbuch III : SGB III), l'indemnité de chômage partiel conjoncturel - Kug - est considérée comme faisant partie intégrante de la politique pour l'emploi. Elle a pour vocation d'éviter phases passagères de réduction d'activité ; elle permet en effet au premier de préserver son effectif de main-d'œuvre qualifiée, et au second de préserver son emploi. Lorsqu'il recourt au Kug, l'employeur continue donc de verser salaire et cotisations sociales puisque le contrat de travail est maintenu. II peut solliciter l'application de ce régime dans le cas d'une baisse de l'activité et des rémunérations, temporaire et inévitable, en raison de difficultés ou d'un événement de caractère exceptionnel, mais aussi en situation de ralentissement conjoncturel.

Le recours à la mesure Kug est accordé à l'entreprise après examen de sa demande par l'agence pour l'emploi dont elle dépend. Les conditions requises sont les suivantes : la baisse de l'activité et des rémunérations doit être supérieure à $10 \%$, et concerner au moins un tiers des salariés. S'y ajoute que, au préalable, toutes les autres possibilités offertes par le droit du travail doivent être épuisées : congés légaux et comptes épargne-temps. Car l'indemnité de chômage partiel conjoncturel n'est pas une mesure de soutien public versée à l'employeur par l'Etat (et financée par l'impôt), mais une prestation sociale versée aux salariés au titre de l'assurance chômage. Les salariés ne peuvent en bénéficier que s'ils occupent un emploi soumis à cotisations sociales et que leur contrat de travail n'est pas interrompu durant la période d'activité partielle. La mesure exclut donc le licenciement et, partant, l'application du régime général des allocations chômage, tout en poursuivant une approche similaire d'une protection assise sur la redistribution. II s'agit donc d'une ultime protection avant tout licenciement économique.

L'indemnité de chômage partiel Kug est calquée sur l'allocation chômage : elle correspond à un montant forfaitaire de $60 \%$ du revenu net pour les salariés sans enfants, à $67 \%$ pour les salariés avec enfants. Si l'employeur ne verse pas de rémunération pour les heures chômées, il continue en revanche de payer les cotisations patronales dues pour ces heures, et il doit également prendre à sa charge les cotisations sociales du salarié pour la période chômée.

Dans le cadre de ses deux programmes conjoncturels, le gouvernement fédéral a apporté quatre modifications transitoires à ce régime, valables pour les seules années 2009 et 2010. L'employeur voit réduite de moitié la part des cotisations sociales du salarié qu'il lui revient de payer pour les heures d'inactivité. II peut aussi en être totalement exonéré à la condition qu'il s'engage à offrir à ses salariés en chômage partiel une formation continue durant les heures chômées - ce qui apporte une modification substantielle à l'approche du dispositif. Quant aux tout licenciement en aidant aussi bien l'employeur que le salarié à surmonter les 
conditions d'accès à cette mesure, elles ont été simplifiées : il suffit désormais de justifier d'une baisse des rémunérations de $10 \%$, quel que soit l'effectif concerné, tandis que le caractère inéluctable de la baisse de l'activité ne fera plus l'objet d'un examen approfondi. Enfin, dernière modification, les travailleurs intérimaires auront pour la première fois un accès limité aux indemnités de chômage partiel s'il y a impossibilité de les employer à brève échéance et que par conséquent leur activité se retrouve momentanément suspendue.

En Allemagne, la durée du chômage partiel est limitée à six mois. Toutefois, lorsque la situation du marché de l'emploi est exceptionnelle, cette période peut être étendue par décret jusqu'à 24 mois. Elle a ainsi été portée à 18 mois au $1^{\mathrm{er}}$ janvier 2009 et s'applique à tous les salariés éligibles au droit à l'indemnisation Kug avant le 31 décembre 2009. La durée de l'indemnisation est la même pour tous les salariés de l'entreprise, indépendamment de leur ancienneté.

L'agence pour l'emploi peut proposer, de manière temporaire, un autre emploi aux bénéficiaires de l'indemnité Kug (deuxième contrat de travail). Ceux-ci ont obligation de se présenter à l'agence pour l'emploi sur convocation de cette dernière et d'accepter une offre d'emploi jugée « acceptable » (zumutbar). Dans le cas d'un refus de se présenter sans motif valable devant le nouvel employeur, le versement de l'indemnité Kug est supprimé dans la plupart des cas sur une période de trois semaines (période de blocage). Le salaire perçu dans le cadre du deuxième emploi venant augmenter les revenus du salarié, celui-ci se voit réduire en conséquence son indemnité Kug. Cette variante n'a pas été modifiée.

\section{Les deux autres régimes d'activité partielle}

Le régime de chômage technique de transfert, qui concerne les restructurations d'entreprises, s'applique même lorsque la réduction d'activité a un caractère durable. Afin d'éviter des licenciements et d'améliorer les perspectives de reclassement des salariés concernés, l'indemnité de chômage partiel de transfert est alors versée sur une période plus longue, mais qui ne peut excéder un an. Les salariés bénéficiaires doivent être alors regroupés au sein d'une entité organisationnelle autonome : il s'agit la plupart du temps d'une « société de transfert » (Transfergesellschaft) qui a pour mission de reclasser les salariés et de les former dans cette perspective. De telles mesures sont en règle générale menées sur la base d'un accord de compensation des intérêts (Interessenausgleich) conclu entre l'employeur et le conseil d'entreprise, et accompagnées d'un plan social portant sur le versement, par l'ancien employeur, d'une indemnité venant en complément de l'indemnité de chômage partiel de transfert.

Une évaluation de ce dispositif par l'Institut d'études prospectives sur l'emploi (Institut zur Zukunft der Arbeit, IZA) a révélé que, contrairement à leur objectif, ces mesures n'avaient pas d'impact positif sur les chances de retrouver un emploi. Néanmoins, les effets négatifs de la réglementation en vigueur avant les réformes Hartz (2003/05) ne sont plus perceptibles. Celle-ci fixait une durée de versement plus longue, allant jusqu'à 24 mois, et ne portait qu'à la marge sur des mesures de qualification et de reclassement (Schneider et al., 2007).

Le régime du chômage partiel saisonnier a, quant à lui, vocation à stabiliser l'emploi dans le BTP en évitant les licenciements en cas de réduction d'activité et du volume des commandes dans les périodes de météo défavorable, c'est-à-dire pendant la saison hivernale. L'indemnité de chômage partiel saisonnier existe sous sa forme actuelle depuis 2006. Elle a alors remplacé « l'indemnité de mauvais temps » (Schlechtwettergeld) et l'aide au secteur de la construction en période hivernale. Désormais, les salariés d'entreprises de construction ainsi que les couvreurs, jardiniers et paysagistes se voient verser, sur une période de mauvais temps dont la durée a été réduite (elle va désormais de décembre à mars), des indemnités pour les réductions d'activité d'ordre économique ou climatique, si ces dernières présentent un caractère temporaire et inévitable.
L'option d'un deuxième emploi

L'indemnité de chômage partiel de transfert

Une mesure qui s'est révélée inadaptée

Le chômage partiel saisonnier, mesure sectorielle réservée au BTP 
Deux types d'indemnités

Un rôle central pour accompagner la transition dans les nouveaux Länder après l'Unité allemande...

... et pour amortir socialement les crises de 1993 et 2002/03
L'indemnité est allouée dès la première heure chômée, mais seulement si la perte d'activité ne peut être compensée par la liquidation des actifs de comptes épargne-temps. Autrement dit, ce régime respecte les accords conventionnels de branche sur la flexibilisation du temps de travail, puisque la compensation des heures chômées par les congés accumulés dans le cadre de comptes épargnetemps a priorité sur le versement d'un revenu de substitution. L'indemnité de chômage partiel saisonnier est financée elle aussi par les cotisations d'assurance chômage. Quant aux salariés du BTP dont le contrat de travail ne peut être résilié pendant la période de mauvais temps pour des raisons d'ordre climatique, ils bénéficient en outre, dans ce système de répartition, d'une «allocation hivernale » (Wintergeld) par heure travaillée.

\section{Le chômage partiel a joué un rôle important en Allemagne lors des crises structurelles ou conjoncturelles}

Dans les années qui ont précédé la crise économique actuelle, le recours au chômage partiel restait une pratique relativement marginale en Allemagne. Toutefois au début des années 1990, cet instrument a joué un rôle central dans le cadre de la restructuration de l'économie de l'ex-RDA : en 1991, on recensait dans les nouveaux Länder en moyenne annuelle plus de 1,6 million de salariés au chômage partiel, dont une grande partie en « activité partielle zéro » (Kurzarbeit null), c'està-dire en arrêt total d'activité. Le système de chômage partiel étant destiné à pallier des réductions d'activité temporaires, il n'a pu atténuer qu'à court terme les suppressions d'emplois dans l'ex-RDA: la plupart des salariés au chômage partiel ont été ensuite redirigés vers le chômage, la retraite anticipée ou d'autres mesures de politique active pour l'emploi.

Lors de la crise économique qui a touché l'Allemagne de l'ouest en 1993, on a pu constater une seconde vague de chômage partiel : elle a touché en moyenne annuelle près de 760000 salariés dans l'industrie manufacturière. Après 1997, le chômage partiel a fortement reculé. Une nouvelle crise conjoncturelle en 2002/03 a conduit à un nouveau pic, de 200000 personnes, suivi d'une baisse progressive jusqu'en 2007 où on ne dénombrait plus que 68000 personnes bénéficiant de ce régime. La flexibilisation du temps de travail négociée dans le cadre de conventions collectives et d'accords d'entreprises, notamment via la multiplication des comptes épargne-temps, a en effet considérablement réduit le recours au système de chômage partiel par les entreprises - du moins jusqu'à récemment.

Le chômage partiel en Allemagne depuis le début des années 1990

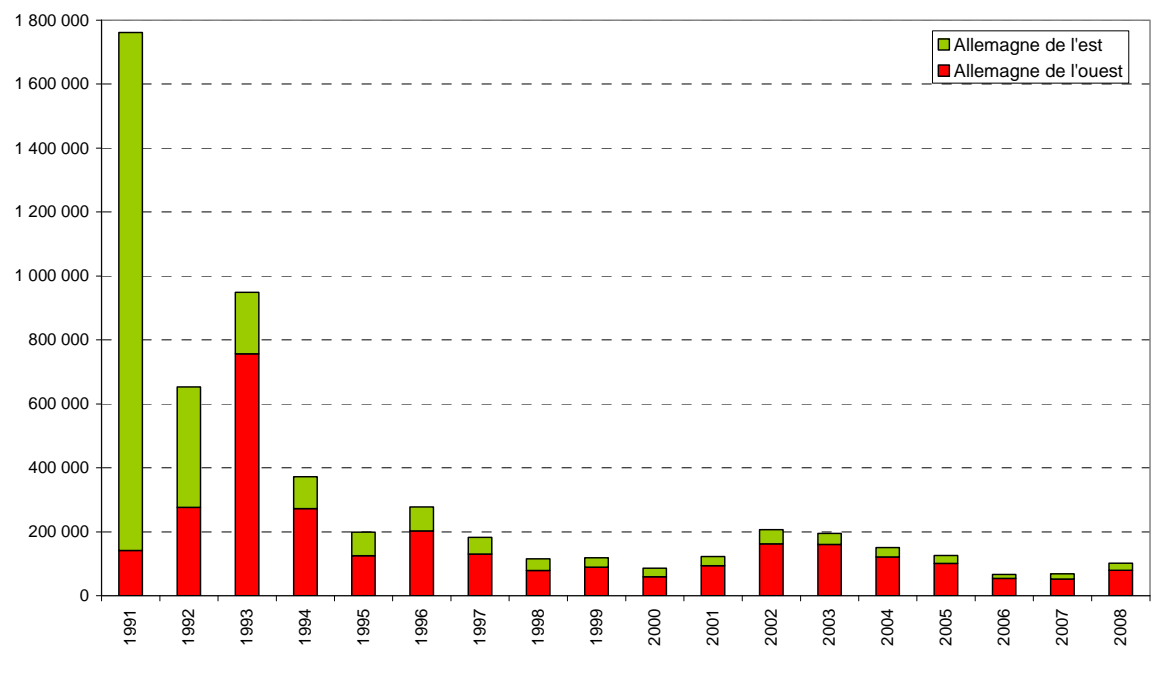

Source : Agence fédérale pour l'emploi 
Evolution du chômage partiel dans la crise économique actuelle (demandes déposées et nombre de salariés concernés en Allemagne de l'ouest et de l'est)

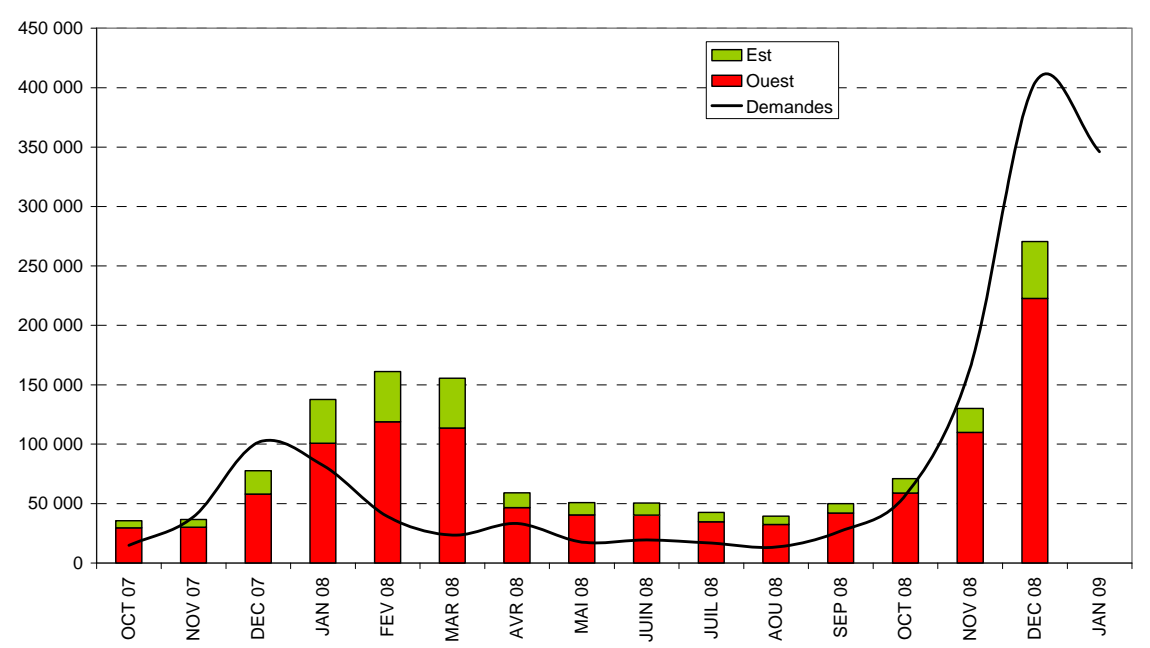

Source : Agence fédérale pour l'emploi

Fin 2008, le nombre de demandes émanant des entreprises s'est à nouveau rapidement multiplié. En décembre 2008, le chômage partiel a été ainsi requis pour 400000 salariés au total, dont les trois quarts (plus de 290000 ) au titre de la mesure Kug, le chômage partiel saisonnier et le chômage partiel de transfert ne concernant respectivement qu'un peu moins de 100000 et 2000 personnes. Le léger recul du nombre de demandes observé en janvier 2009 est à attribuer exclusivement au chômage partiel saisonnier (passé à moins de 50000 personnes), tandis que le nombre de demandes de chômage partiel conjoncturel est resté au même niveau. Etant donné que le nombre de salariés au chômage partiel est calculé avec un certain décalage dans le temps, les informations postérieures au début de la crise qui affecte l'économie réelle crise ne sont pas encore disponibles. Cependant, l'évolution du volume des demandes laisse entrevoir une forte hausse du nombre de salariés bénéficiaires du régime Kug.

Nombre de salariés faisant l'objet de demandes Kug en décembre 2008 - par secteurs d'activités -

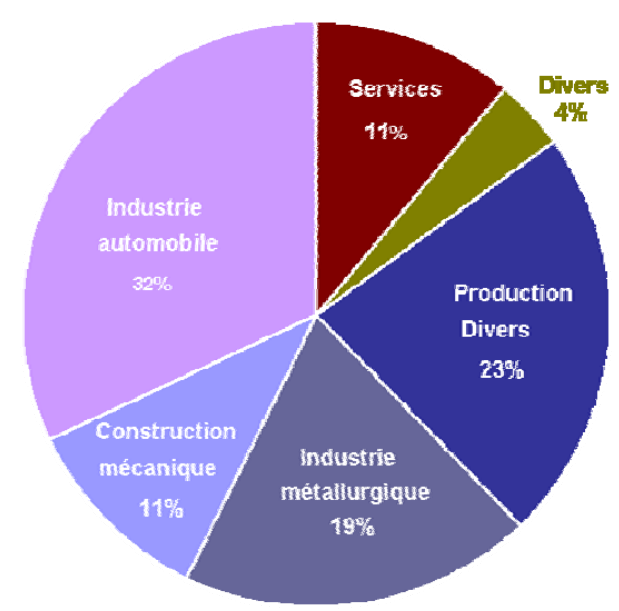

Source : Agence fédérale pour l'emploi

A en croire les médias, le chômage partiel touche principalement les grandes entreprises industrielles, ce que les données disponibles en la matière ne confirment toutefois qu'en partie. Les résultats du panel d'entreprises de l'Institut für Arbeitsmarkt- und Berufsforschung, Institut de recherche sur l'emploi de l'Agence
Un recours accru au chômage partiel depuis la fin 2008 ... ...essentiellement dans le secteur manufacturier 
Les PME sont largement concernées

Dans l'ensemble, pas d'arrêt total de l'activité jusqu'ic

Une forte hausse des dépenses d'indemnisation est prévue

Une régulation de l'emploi qui limite la réactivité au marché...

.. mais qui stimule la compétitivité technologique... fédérale pour l'emploi (IAB), parus en 2003 - c'est-à-dire lors du dernier recours massif à ce régime pour des raisons conjoncturelles - attestent la prédominance du secteur secondaire : près de $60 \%$ de l'ensemble des bénéficiaires relevaient de l'industrie, et seulement près de $20 \%$ du secteur des services (Deeke, 2005). La crise conjoncturelle actuelle n'a pas modifié cette répartition : les demandes de chômage partiel de décembre 2008 émanent presque exclusivement de l'industrie manufacturière, principalement des constructeurs automobiles et de leurs sous-traitants ; un dixième seulement des salariés concernés est issu du secteur des services. L'indemnité de chômage partiel Kug apparaît dès lors comme un instrument quasi fait sur mesure pour les besoins de l'industrie.

La répartition des demandes par taille d'entreprise montre que le chômage partiel (Kug) ne se limite nullement aux grands groupes. Bien que la proportion des bénéficiaires soit la plus élevée dans les sociétés de plus de 500 salariés (en 2003, elle était de plus de $5 \%$ ), près des trois quarts du chômage partiel, tous régimes confondus, se concentre dans les entreprises de moins de 20 collaborateurs (Deeker, 2005). Même si les grandes entreprises sont surreprésentées au regard de leur importance relative, une part significative revient donc aux PME.

La moyenne de la période d'inactivité déclarée par les entreprises dans le cadre du régime Kug ne permet pas d'en conclure à un impact visible de la crise sur l'activité. En septembre 2008, cette moyenne n'était que de $49 \%$ du total des heures habituellement travaillées alors qu'elle atteignait $55 \%$ l'année précédente. On peut dès lors en conclure que, actuellement en tout cas, les cas d'arrêt total de l'activité sont plutôt rares. Néanmoins, un certain nombre d'experts prévoient un recul accentué de l'activité à la mi-2009, qui pourrait alors se traduire par une vague de licenciements, les employeurs ne faisant généralement usage du chômage partiel que durant six mois en moyenne.

Par ailleurs, les dépenses relatives aux indemnités Kug ne révèlent pas encore de hausse notoire pour 2008, sachant toutefois que le budget du dernier trimestre 2008 n'a pas encore été publié par l'Agence fédérale pour l'emploi (ses missions sont comparables en France à un guichet unique réunissant l'ANPE et les Assedic). Or sous le double impact des réformes du régime du chômage partiel comme des perspectives négatives pour l'évolution conjoncturelle, on devrait assister à une forte hausse des dépenses liées à ce régime dans les mois à venir. L'Agence fédérale pour l'emploi a annoncé en prévision qu'elle allait porter le montant des fonds alloués au chômage partiel de 300 millions à 1,4 milliard $€$ en 2009, puis à 1,2 milliard $€$ en 2010. A quoi s'ajoutent 400 millions $€$ supplémentaires consacrés chaque année aux mesures de qualification.

\section{Un outil de flexibilité dans le cadre de la politique pour l'emploi}

Comme en France et dans d'autres pays d'Europe continentale, le marché de l'emploi allemand se caractérise par la forte intensité de sa régulation. Dans leur processus d'adaptation à la situation économique, les entreprises allemandes sont donc soumises à des conditions cadre plus restrictives que dans les pays anglo-saxons, à la réglementation plus souple. Les seuils salariaux minimum négociés sur une base contractuelle par les partenaires sociaux, et aujourd'hui fixés par la loi dans un nombre croissant de cas, ne permettent pas d'abaisser les salaires en réaction à l'évolution du marché. Par ailleurs, en raison d'une protection contre le licenciement stricte en comparaison, les entreprises ne peuvent, pour adapter leur effectif à l'état des carnets de commandes, recourir au licenciement que sous condition.

Cette organisation plutôt rigide est souvent considérée comme la cause du niveau relativement élevé de chômage en Allemagne. D'un autre côté, elle permet aux entreprises exportatrices de préserver leur avantage compétitif dans des secteurs à forte intensité technologique. Car la garantie institutionnelle de la durée ainsi apportée aux contrats de travail stimule l'investissement dans la formation 
ciblée d'une main-d'œuvre hautement qualifiée, condition sine qua non de la qualité comme de la réputation de la production allemande.

Face aux rigidités institutionnelles du marché de l'emploi, les mesures de promotion de la "flexibilité interne » représentent pour les entreprises allemandes une ressource stratégique leur permettant de pallier les variations conjoncturelles. II s'agit en premier lieu des divers modèles de flexibilisation du temps de travail. Ces dernières années, la généralisation des comptes épargne-temps constitués sur le long terme a ainsi permis aux entreprises de mieux compenser pics de production et recul des commandes. De même, la multiplication des accords d'entreprise (Schrœder, 2005) leur a apporté la souplesse requise pour ajuster le volume des heures travaillées aux variations des entrées de commandes. Qu'ils s'accompagnent ou non de compensations salariales, ces accords apportent une flexibilisation considérable de la rémunération.

Au total, les entreprises disposent donc, via leur flexibilité interne, d'instruments propres aux fonctions comparables à celles des licenciements collectifs. Quant au régime de chômage partiel, il peut être considéré comme un soutien apporté par la politique de l'emploi à ces stratégies de flexibilisation. Car cette mesure vient en complément ultime d'un dispositif à plusieurs étages visant à éviter les licenciements : elle n'intervient qu'après que droits à congés, comptes épargne-temps des salariés, etc. sont épuisés - ce qui, rappelons-le, est la condition sine qua non pour le recours au chômage partiel.

L'avantage du chômage partiel réside donc dans son effet stabilisateur, à la fois pour chaque entreprise considérée isolément et pour le système productif allemand dans son entier. Et ce régime ne se traduit pas mécaniquement par une hausse des dépenses du système d'assurance chômage, puisque le coût de la prestation est moins élevé qu'en cas de chômage. En outre, les conseils d'entreprise et les syndicats sont dans l'ensemble favorables au chômage partiel dans la mesure où il contribue à empêcher les licenciements, à maintenir dans l'entreprise les salariés ayant de l'ancienneté et que, en règle générale, il n'est instauré que pour une courte durée et s'accompagne d'une baisse limitée des revenus. Enfin, ce n'est que lorsqu'aucune amélioration de la situation de l'entreprise n'a pu être constatée au bout de plusieurs mois de chômage partiel que l'employeur procède à une restructuration qui s'accompagne alors de licenciements et du recours aux transferts prévus dans le cadre de l'assurance chômage.

L'indemnité de chômage partiel peut par ailleurs revêtir une fonction de politique sociale lorsque, comme cela était envisagé dès l'origine, la période d'inactivité des salariés est consacrée à la formation continue, ce qui accroît l'employabilité du salarié. In fine, le chômage partiel se révèle ainsi un mécanisme de protection accrue contre le chômage. Et dans une certaine mesure contre la précarité puisque, dans le régime transitoire 2009/10, les travailleurs intérimaires (au demeurant peu protégés) peuvent eux aussi bénéficier des indemnités de chômage partiel qui leur offrent une sécurité en matière de rémunération dans les périodes entre deux missions, quoique dans la pratique, il se révèle difficile pour eux de faire valoir leurs droits. Car pour qu'un travailleur intérimaire puisse prétendre à ce régime, il faut pouvoir justifier de manière crédible d'une perte totale d'activité prévisible sur une période de plus de trois mois (et non d'une réduction partielle de l'activité, comme c'est le cas pour les salariés occupant un emploi 'régulier'). Les entreprises de travail temporaire doivent ainsi prouver qu'il n'existe pas de possibilité pour une mission dans d'autres branches que la leur ou, le cas échéant, proposer à leur employé une reconversion « acceptable » (zumutbar).

On peut cependant aussi considérer le chômage partiel sous un angle plus critique. Son effet conservateur sur la structure de l'emploi tend en effet à handicaper le processus de mutation structurelle de l'activité, en particulier lorsque le chômage partiel est octroyé sur une longue période, en cas d'arrêt total de l'activité («activité partielle zéro») ou de changements d'ordre plus structurel que conjoncturel. ...et incite à la flexibilité intra-entreprise

Chômage partiel : un outil politique d'appui à la flexibilisation du travail

Effet structurellement stabilisateur pour le système productif ...

... et pour le marché de l'emploi

Mais un système qui freine aussi la mutation des activités ... 
... et dont la nouvelle configuration pourrait avoir des effets pervers
La prolongation de la durée de versement de l'indemnité Kug jusqu'à 18 mois, décidée dans le cadre des plans de relance actuels, est susceptible d'accentuer cette tendance, bien qu'il soit peu probable que les employeurs utiliseront la totalité de cette période : selon les données de l'Agence fédérale pour l'emploi, la durée moyenne se situe entre trois et quatre mois. II n'existe pas moins le risque que l'emploi soit maintenu de manière artificielle dans des secteurs moins productifs et innovants, au détriment d'un redéploiement vers des activités plus efficientes. Or le régime du chômage partiel de transfert, qui a justement pour vocation de faciliter le reclassement professionnel des salariés, n'a pas pu démontrer de manière significative jusqu'ici d'effets positifs. Ensuite, un recours de longue durée et plutôt passif au chômage partiel et à ses revenus de transfert risque aussi de se transformer en passerelle vers la retraite anticipée.

Ce sont LeS EFFETS StabILISATEURS INDÉNIABLES DU CHOMAGE TECHNIQUE qui ont incité le gouvernement fédéral à faciliter l'accès à ce régime dans le cadre de ses deux programmes conjoncturels. Et de fait, ce régime semble amortir pour l'instant les risques d'impact social de la récession. Mais il convient de se demander aussi si les incitations contenues dans le nouveau régime du chômage partiel ne pourraient pas se révéler problématiques par certains aspects. Car l'exonération totale de la part patronale des cotisations sociales pourrait pousser les entreprises, pour des raisons de coûts, à proposer des mesures de formation à bas prix et peu judicieuses - ce qui ne serait pas sans conséquence sur le niveau global des qualifications. De même, via l'allongement de la durée de versement et les allègements favorables aux entreprises, les réformes ont globalement augmenté de manière significative le coût de ce système. Une crise prolongée, s'accompagnant de licenciements massifs, représenterait alors un risque considérable pour l'équilibre financier du système allemand d'assurance chômage, puisqu'il s'agira pour ainsi dire de financer une protection 'doublée' sur une durée pouvant atteindre jusqu'à trois ans : 18 mois de Kug, puis 12 mois d'allocations chômage (et même 24 pour les plus de 50 ans, soit 42 mois au total).

II faut en outre garder à l'esprit que le chômage partiel ne bénéficie dans les faits qu'à une certaine partie des salariés et des entreprises. Or le marché de l'emploi allemand est marqué par un fort clivage entre d'un côté des contrats de travail dits « classiques » et, de l'autre, diverses formes d'emploi atypiques. Le chômage technique s'applique d'une manière typique à la main-d'œuvre industrielle occupant un emploi soumis à cotisations sociales, et qui, par définition, appartient à ce premier groupe qu'il convient de considérer comme privilégié. En d'autres termes : le régime de l'activité partielle s'adresse ainsi majoritairement aux « insiders ", c'est-à-dire à ceux qui ont un emploi stable et bénéficient d'une protection sociale optimale. II risque donc de creuser encore plus un clivage déjà prononcé en comparaison européenne. Enfin, si on considère le fait que le régime de chômage partiel est certes financé par tous les acteurs - salariés et employeurs cotisant à l'assurance chômage, mais que n'y recourt qu'un nombre limité de branches, alors le chômage partiel s'assimile à un considérable transfert social des services au profit de l'industrie.

Traduction : I. Bourgeois et S. Hazouard

\section{Indications bibliographiques}

-DEEKE A., "Kurzarbeit als Instrument betrieblicher Flexibilität. Ergebnisse aus dem IAB-Betriebspanel 2003 », IAB Forschungsbericht, IAB, Nuremberg, 12/2005,

- SCHNEIDER, HiLmAR et AL., "Evaluation der Maßnahmen zur Umsetzung der Vorschläge der Hartz-Kommission », Module 1b: Förderung beruflicher Weiterbildung und Transferleistungen, IZA, DIW, infas, Bonn, 2007

- SCHRCEDER W., "Le modèle syndical allemand n'existe plus ", in BouRgeols I. (dir.), Le modèle social allemand en mutation, Travaux et Documents du CIRAC, Cergy-Pontoise, 2005. 